\title{
LINEARLY QUASI-ORDERED COMPACT SEMIGROUPS
}

\author{
N. J. ROTHMAN
}

By semigroup, we mean a Hausdorff topological space together with a continuous associative multiplication. We are concerned here with the existence of local subsemigroups, which are arcs, in compact connected semigroups whose principal left ideals are linearly ordered by $C$. In addition, we seek local cross sections at certain idempotent elements in such semigroups.

In [5], Koch has shown that if $S$ is a compact connected semigroup with identity, then $S$ contains an arc. In [7], Mostert and Shields obtained local one parameter semigroups at the identity. Hunter, in [2], and Hunter and Rothman, in [3], have obtained arcs which are local subsemigroups and certain local cross sections in compact connected abelian topological semigroups. Our approach is similar to that in [2] and [3], and concerns noncommutative semigroups.

We follow the notation and terminology of $[1 ; 2 ; 3 ; 11]$. In particular, a nonempty subset $L(R, I)$ of a semigroup $S$ is a left (right, two sided) ideal of $S$ if $S L \subset L(R S \subset R, S I \cup I S \subset I)$. The left ideal $L$ is principal if for some $x \in L, L=\{x\} \cup S x$. We denote by $L_{p}$ the set of those $x$ in $S$ for which $\{x\} \cup S x=\{p\} \cup S p$. The symbol $K$ is reserved for the minimal ideal of a semigroup (when $S$ is compact, $K$ exists), and $E$ is used to denote the set of idempotent elements of $S$ (when $S$ is compact, $E$ is nonvoid). If $e \in E$, the maximal subgroup of $S$ containing $e$ is designated by $H_{e}$.

It is known that the sets $L_{p}$ form an upper semi-continuous decomposition of $S$, when $S$ is compact. We let $S^{\prime}$ be the associated hyperspace of this decomposition and $\phi$ be the natural mapping. That is $\phi: S \rightarrow S^{\prime}$ is given by $\phi(x)=\left\{L_{x}\right\}$. We are interested in the cases when $S^{\prime}$ is again a semigroup and $\phi$ is a homomorphism. In particular, Theorem 1 gives necessary and sufficient conditions that $S^{\prime}$ be a standard thread [1].

Definition. A semigroup $S$ is said to be left linearly quasi-ordered if for each $x$ and $y$ in $S$, either $\{x\} \cup S x \subset\{y\} \cup S y$ or $\{y\} \cup S y \subset\{x\}$ $\cup S x$.

It is easy to see that the order induced on $S, x \leqq y$ if and only if $\{x\} \cup S x \subset\{y\} \cup S y$, is a continuous quasi-order in the sense of Nachbin [8] and Ward [10]. It follows that each compact subset of $S$ has a maximal element.

We assume for the remainder of this note, that $S$ is a compact

Received by the editors May 12, 1961. 
connected semigroup. Since we will concern ourselves only with left linearly quasi-ordered semigroups, we omit the term left. We note that the right linearly quasi-ordered semigroups are dual to the left ones when $x y=y x$.

Lemma 1. Let $S$ be a linearly quasi-ordered semigroup. Then (i) $S^{2}$ $=S$, (ii) for each closed left ideal $L$ of $S$ and $y \in S-L, L \subset S y$, (iii) $S$ $=E S=S E=E S E$, and (iv) for each $x \in S, x S \subset S x$.

Proof. (i) Let $y \in S-S^{2}$, then $y \in S-S x$ for all $x \in S$. If $x \neq y$, then $\{x\} \cup S x \subset S y$ by the linear quasi-order, and hence, $x \in S y$. It follows that $S^{2}=S-\{y\}$ is open and closed in $S$, but $S$ is connected and $S^{2}$ is nonvoid. This is a contradiction, hence, $S^{2}=S$.

(ii) Let $L$ be a closed left ideal of $S$ and $y \in S-L$. For each $x \in L$, $\{x\} \cup S x \subset L$; hence $\{x\} \cup S x \subset\{y\} \cup S y$ by the linear quasi-order and $x \in S y$, that is $L \subset S y$.

In order to obtain (iii) and (iv), we show that $e S \subset S e$ for each $e \in E$.

Let $e \in E$ with $e S \nsubseteq S e$. For $y \in e S-S e, S e \subset S y$ by (ii). Now $e$ and $y$ belong to $S e S$, hence by a result of Koch [4, Corollary 2 to Theorem 3], $S e$ is maximal among [Sx: $x \in S e S]$, hence, $S y \subset S e$. From this contradiction, it follows that $e S \subset S e$.

(iii) We show first that $S=S E$. If $y \in S-S E$, then for each $e \in E$, $e \in S y$ and hence, $S e S \subset S y S$. Now $S=S E S$ by [6, Corollary 1] and $S=S E S \subset S y S$. Hence $y=a y b$ for some $a$ and $b$ in $S$. Thus, there is an $f \in E$ such that $y f=y[9]$ and $S=S E$.

In order to see that $S=E S$, we note that for each $f \in E, S f$ $=U\left[e S: e \in L_{f} \cap E\right] ; \quad$ since, $\quad S\left(U\left[e S: e \in L_{f} \cap E\right]\right) \subset S f S \subset S f \quad$ and $S\left(\mathrm{U}\left[e S: e \in L_{f} \cap E\right]\right)$ is a left ideal containing $f$. Now, $S=S E$ $=\bigcup[S f: f \in E]=\bigcup\left(U\left[e S: e \in L_{f} \cap E\right]: f \in E\right)=E S$. The equations $E S E=E(S E)=E S=S$ establish the last equality.

(iv) Let $x \in S$ and $y \in x S-S x$. Then $S x \subset S y$ and both $x$ and $y$ belong to $S x S$ by (iii). Again, we apply the result of Koch as above and find that $S y \subset S x$. Hence, $x S \subset S x$.

We observe that for each $x \in S,\{x\} \cup S x=S x$ and that $S x$ is a two sided ideal in $S$. In fact, we have

Corollary 1. Each closed left ideal in $S$ is a principal left ideal and a two sided ideal.

Proor. Let $L$ be a closed left ideal of $S$. Since the quasi-order, $x \leqq y$, is continuous, $L$ has a maximal element $x$. Now $S x \subset L$ and for each $y \in L, S y \subset S x$; hence $S x=L$. Since $x S \subset S x, S x S \subset S x$ and $S x$ is a two sided ideal. 
Corollary 2. The minimal ideal $K$ is a minimal left ideal.

THEOREM 1. Let $S^{\prime}$ be the hyperspace of the upper semi-continuous decomposition of $S$ by the sets $L_{p}$. Then $S^{\prime}$ is a semigroup with the multiplication $\left\{L_{x}\right\}\left\{L_{y}\right\}=\left\{L_{x y}\right\}$, and a standard thread if and only if $S$ is linearly quasi-ordered.

Proof. Let $S^{\prime}$ be a standard thread and let $x$ and $y$ belong to $S$ with $\phi(x) \leqq \phi(y)$. Then, either $L_{x}=L_{y}$ or there is an element $b$ in $S$ with $\phi(x)=\phi(b) \phi(y)$ and $L_{x}=L_{b y}$. Thus $S x=S y$ or $S x=S b y \subset S y$ and $S$ is linearly quasi-ordered.

On the other hand, let $S$ be linearly quasi-ordered. Since each closed principal left ideal is an ideal, the upper semi-continuous decomposition of $S$ by the sets $L_{p}$ is the same as the upper semi-continuous decomposition of $S$ by the sets $J_{p}\left(x \in J_{p}\right.$ if and only if $\{x\} \cup S x \cup x S$ $\left.\cup S x S=\{p\} \cup S_{p} \cup p S \cup S p S\right)$. In order to show that $S^{\prime}$ is a semigroup, it suffices that $(S x)(S y)=S x y$. Now $S x S=S x$, hence, $S x S y$ $=S x y$ by associativity. Since $\phi$ is a continuous homomorphism, $S^{\prime}$ is a compact connected semigroup with zero $(=\phi(K))$ and identity (= $\phi(e)$, where $S=S e$ ). The order on $S^{\prime}$ induced by that on $S$ is total and open intervals are open sets. Hence, $S^{\prime}$ has the order topology and thus is a standard thread.

For the remainder of this paper, we will assume that $S^{\prime}$ is a standard thread. The following lemma is in part a generalization of a result of Hunter [2, Lemma 2], and utilizes his proof.

Lemma 2. Let $T$ be a subsemigroup of $S^{\prime}$. Suppose that $T$ contains only two idempotent elements and is a standard thread from $\phi(e)$ to $\phi(f)$, where $e$ and $f$ belong to $E$. Then $\phi^{-1}(T)$ contains a compact connected subsemigroup $N$ such that $N$ modulo $\left(N \cap L_{e}\right)$ is a standard thread from $\left\{N \cap L_{e}\right\}$ to $f$ homeomorphic and isomorphic to $T$, and $f S-S e$ $=H_{f}\left(N-L_{e}\right)$.

Proof. We restrict our attention to the subsemigroup $f S$ in $S$. It is easily seen that $f S$ is a compact connected linearly quasi-ordered semigroup with identity $f$. Let $R=\left(\phi^{-1}(T) \cap f S\right)$ modulo $\left(L_{e} \cap f S\right)$. We first show that $R$ is connected. If $R=A \cup B$, where $A$ and $B$ are mutually exclusive compact sets, with $\left\{L_{e} \cap f S\right\} \in B$, then, since $A$ is compact, there is an $x \in A$ such that $\phi(x)$ is the first point of $\phi(A)$ in $T$ in the order from $\phi(e)$ to $\phi(f)$. (We are identifying $\left(\phi^{-1}(T) \cap f S\right.$ ) $-\left(L_{e} \cap f S\right)$ and $R-\left\{L_{e} \cap f S\right\}$.) Let $V$ be an open set such that $A \subset V$ and $V \cap B=\square$. For any open set $U$ containing $f$, there is a $t \in R$ such that $L_{t} \cap U \neq \square, L_{t} \neq L_{f}$. For, let $b \in L_{f} \cap f S=H_{f}$, let $b^{-1}$ be the inverse of $b$ in $H_{f}$, and let there be sets $L_{p}$ arbitrarily close to $b$. 
The sets $L_{p b}{ }^{-1}$ are then arbitrarily close to $f$. By continuity of multiplication, there is an open set $W$ containing $f$ such that $x W \subset V$. Since $x f=x, x \in x W$ and there is an $L_{t}$ such that $t \in L_{t} \cap W$ and $\phi(t)<\phi(f)$. Since $x L_{t} \subset L_{x t}, L_{x t} \cap V \neq \square$ and $x t \in A$. Since $T$ has only two idempotent elements, $\phi(x t) \neq \phi(x)$. Since $\phi(x t) \leqq \phi(x), \phi(x t)<\phi(x)$ and $\phi(x)$ is not the minimal element of $\phi(A)$. This contradiction shows that $R$ is connected, it is clearly compact and hence a continuum. The semigroup $R$ has a zero $\left\{L_{e} \cap f S\right\}$ and an identity $f$ and no other idempotent elements. It follows from [7] that there is a standard thread $P$ from the zero of $R$ to the identity of $R$. Letting $\delta$ be the natural mapping of $\phi^{-1}(T) \cap f S$ onto $R$ and $J=\delta^{-1}(P-\{0\})$, we see that $N=\bar{J}$ is the desired semigroup.

Let $x \in f S-S e$ and $n \in N \cap L_{x}$, then $x \in f S n$ and hence $x=f t n$. But $\phi(x)=\phi(n)=\phi(f) \phi(t) \phi(n)$ and thus $\phi(f t)=\phi(f)$. Hence $f t \in H_{f}$ and $x \in H_{f}\left(N-L_{e}\right)$. It follows that $f S-S e=H_{f}\left(N-L_{e}\right)$.

For use in the next lemma, we note that if $e$ and $f$ belong to $E$ with $L_{e}=L_{f}$ then $e f=e$ and $f e=f$.

Lemma 3. If $f \in E$, then, for each $e \in L_{f} \cap E$, eS is topologically and algebraically isomorphic to $f S$ under the mapping ex $\rightarrow$ fex.

Proof. Let $\theta: e S \rightarrow f S$ be defined by $\theta(x)=f x$. If $\theta(x)=\theta(y)$, then $f x=f y$ and $e f x=e f y$ but then $x=e x=e y=y$ and $\theta$ is injective. It is clear that $\theta$ is surjective and by its definition continuous, hence $\theta$ is a homeomorphism. Now $\theta(x y)=f x y=f x e y=f x e f y=f(x e) f y=f x f y$ $=\theta(x) \theta(y)$ and $\theta$ is a homomorphism.

Theorem 2. Let $T$ be a subsemigroup of $S^{\prime}$. Suppose that $T$ contains exactly two idempotent elements $g^{\prime}$ and $f^{\prime}$ and that $T=\left[g^{\prime}, f^{\prime}\right]$ is a standard thread. Then, for each $f \in \phi^{-1}\left(f^{\prime}\right) \cap E$, there is a compact connected subsemigroup $N(f) \subset f S$ such that $N(f)$ modulo $\left(N(f) \cap \phi^{-1}\left(g^{\prime}\right)\right)$ is a standard thread isomorphic to $T$, and $S f-S g=L_{f}\left(N(f)-\phi^{-1}\left(g^{\prime}\right)\right)$, where $g \in \phi^{-1}\left(g^{\prime}\right)$.

Proof. The first part of the theorem is a restatement of Lemma 2. We let $f$ be a fixed idempotent element in $\phi^{-1}\left(f^{\prime}\right)$ and $N(f)$ the compact connected semigroup of Lemma 2. For $e \in L_{f} \cap E$, we choose $N(e)=e N(f)$ by Lemma 3 .

Let $y \in S f-S g$, then $y \in e S$ for some $e \in L_{f} \cap E$. Hence $y=p n$, where $p \in H_{\varepsilon}$ and $n \in N(e)-\phi^{-1}\left(g^{\prime}\right)$ by Lemma 2 . Now $p f=p$, so that $y=p f n$ and $y \in L_{f}\left(N(f)-\phi^{-1}\left(g^{\prime}\right)\right)$, since $f n \in f N(e)=f e f N(f)=N(f)$ and the conclusion follows.

CoROllaRy. Under the same hypotheses as in Theorem 2, for each $x \in \phi^{-1}(T)-\phi^{-1}\left(g^{\prime}\right), L_{x}=L_{f} x$. 
Proof. We note that $N(f)$ meets each $L$ set in at most one point and the $L$ sets are disjoint and fill up $\phi^{-1}(T)-\phi^{-1}\left(g^{\prime}\right)$. Since $\phi^{-1}(T)$ $-\phi^{-1}\left(g^{\prime}\right)=L_{f}\left(N(f)-\phi^{-1}\left(g^{\prime}\right)\right)$, the conclusion follows.

For the remainder of this note, we continue to use the hypotheses and notation of Theorem 2. Let us consider the mapping $\delta: L_{f} \times N(f)$ $\rightarrow S f$ given by $\delta(p, n)=p n$. If we restrict $\delta$ to $\left(L_{f} \cap f S\right) \times N(f)$, then $\delta(p, n) \in f S$. Now, $L_{f} \cap f S=H_{f}$ is a compact topological group with identity $f$. If $x$ and $y$ belong to $N(f)$ and $g \in H_{f}$; then $g x=g y$ implies $x=y$, since $N(f)$ meets each $L$ set in at most one point. Let $V$ be a neighborhood of $f$ in $f S$ such that, if $x \in V \cap N(f)$ and $p$ and $q \in V \cap H_{f}$, with $p x=q x$, then $p=q$. Then, for any $a, b$ and $c$ in $V$ with $a b=a c$, $b=c$. We see that if such a $V$ exists, then there is an open set $W$ of $H_{f}$ and an arc $[x, f] \subset N(f)$ such that $\delta \mid \bar{W} \times[x, f]$ is injective. Further, $\delta \mid W \times(x, f]$ is a homeomorphism. We have then

THEOREM 3. There is a local cross section at $f$ in $f S$ if there is a local cancellation semigroup containing $f$ in its interior.

TheOREM 4. If $H_{f}$ is a Lie group, then there is an arc $(x, f]$ in $N(f)$ such that $f S-\left(\phi^{-1}[0, x]\right) \approx H_{f} \times(x, f]$.

Proof. Let $H_{f}$ be a Lie group, then, there is an $x \in N(f)$ such that for all $y \in N(f)-x N(f)$ and $p \in H_{f}, p y=y$ implies $p=f$. Hence, $\delta \mid H_{f} \times(x, f]$ is injective. Using the continuity of the decomposition $\mathcal{L}$, and the compactness of $H_{f}$, it follows that $\delta \mid H_{f} \times(x, f]$ is a homeomorphism.

If each $H e$, for $e \in L_{f}$, is a Lie group, it does not follow that there is an $\operatorname{arc}(x, f]$ such that $S f-S x \approx L_{f} \times(x, f]$, for consider

ExAmple 1. Let $C$ denote the Cantor set with multiplication $x y=x$. Let $I_{1}$ be the usual unit interval and consider $C \times I_{1}$. If $D$ is the upper semicontinuous decomposition of $C \times I_{1}$ for which $C \times I_{1} / D$ is the Cantor fan then this is again a semigroup and there are no local cross sections at the idempotent elements of the form $(x, 1)$. However since each $L_{f} \cap f S=\{f\}$ the arcs $N(f)$ are usual unit intervals and the cross sections trivial.

However, we have the

Corollary. If, for each $e \in E, L_{e}$ is a Lie group, then there is a cross section, $L_{e} \times(x, e]$, at each idempotent element $e$ for which $\phi(e)$ is the right hand end point of a unit or nil thread in $S^{\prime}$.

Proof. This follows directly from Theorems 2 and 4.

We did not consider the compact connected idempotent subsemigroups of $S^{\prime}$ due to 
EXAmple 2. Let $R_{1}$ denote the reals modulo 1 and $I_{3}$ the idempotent semigroup on the unit interval with multiplication given by $x \cdot y$ $=\min (x, y)$. Let $T=R_{1} \times I_{3} \times I_{3}$ and let $S$ be the subsemigroup of $T$ given by $S=\left(R_{1} \times I_{3} \times\{0\}\right) \cup\left(\{0\} \times\{0\} \times I_{3}\right)$. Then $S$ is a linearly quasi-ordered semigroup and $S^{\prime}$ is isomorphic to $I_{3}$. However, the geometric realization of $S$ as a cylinder with a free arc attached shows that there are connected idempotent subsemigroups $P$ in $S^{\prime}$ such that $\phi^{-1}(P) \neq L_{f} N(f)$. Here the $N(f)$ exist by $[2$, Lemma 1$]$.

Further examples can be found in [2] and [3].

\section{REFERENCES}

1. A. H. Clifford, Connected ordered topological semigroups with idempotent endpoints. I, Trans. Amer. Math. Soc. 88 (1958), 80-98.

2. R. P. Hunter, Certain upper semi-continuous decompositions of a semigroup, Duke Math. J. 27 (1960), 283-289.

3. R. P. Hunter and N. J. Rothman, Characters and cross sections for certain semigroups, Duke Math J. (to appear). 401.

4. R. J. Koch, On monothetic semigroups, Proc. Amer. Math. Soc. 8 (1957), 397-

5. - Arcs in partially ordered spaces, Pacific J. Math. 9 (1959), 723-728.

6. R. J. Koch and A. D. Wallace, Maximal ideals in compact semigroups, Duke Math. J. 21 (1954), 681-686.

7. P. S. Mostert and A. L. Shields, One parameter semigroups in a semigroup, Trans. Amer. Math. Soc. 96 (1960), 510-517.

8. L. Nachbin, Topologia e Ordem, Univ. of Chicago Press, Chicago, 1950.

9. K. Numakura, On bicompact semigroups, Math. J. Okayama Univ. 1 (1952), 99-108.

10. L. E. Ward, Jr., Partially ordered topological spaces, Proc. Amer. Math. Soc. 5 (1954), 144-161.

11. A. D. Wallace, The structure of topological semigroups, Bull. Amer. Math. Soc. 61 (1955), 95-112.

The UNIVERsity OF Rochester 